\title{
Experimental Investigations on the Tuning of Active Gate Drivers under Load Current Variations
}

\author{
Erica Raviola, Franco Fiori \\ Electronics and Telecommunication Dpt. (DET) \\ Politecnico di Torino \\ Turin, Italy \\ \{erica.raviola, franco.fiori\}@polito.it
}

\begin{abstract}
Active Gate Drivers have gained of interest as they allow one to shape the switching waveforms finely, thus reducing overshoots and oscillations. However, when fast power switches are exploited, the tuning of such drivers is still challenging. This paper investigates the adjustment of gate current profile under load variations, which is a crucial issue when targeting practical applications. Indeed, a technique, based on the stretching of time intervals, is proposed and its effectiveness, in terms of undershoot reduction, is experimentally assessed.
\end{abstract}

Keywords-Active Gate Driver (AGD), switching waveforms, AGD tuning, power transistors.

\section{INTRODUCTION}

In recent years, the design of power modules featuring higher efficiency has been achieved by the exploitation of faster power switches, amongst which $\mathrm{SiC}$ and $\mathrm{GaN}$ transistors. Indeed, the former outperforms traditional $\mathrm{Si}$ transistors in terms of on-resistance and thermal reliability, the latter in terms of lower parasitic capacitances [1]. As the use of such devices is spreading among practitioners, some drawbacks should be carefully taken into account. Faster devices imply higher $\mathrm{dV} / \mathrm{dt}$ and dI/dt, which worsen the Electro-Magnetic Interference (EMI) performance of power modules [2]. Over-voltages and over-currents are also more prone to occur, thus affecting the reliability of the system [3]. Finally, resonant circuits comprising the inductive and capacitive parasitics of layout, packaging and of active devices themselves may be triggered, resulting in unwanted oscillations superimposed onto the switching waveforms.

Active Gate Drivers (AGDs) are currently being investigated to address the aforementioned issues, as they allow to shape the switching waveforms by modulating the driver strength during transients [4]. Indeed, the modulation can be achieved by modifying the current/voltage/resistance value, and the profile can be fixed, modified during the transient or adapted cycle-by-cycle to account for operating condition variations [5]. With respect to traditional solutions, e.g., snubbers and ferrite beads, AGDs allow a better trade-off between switching power losses and overshoot reduction as the power transistor is slowed down only for a part of the overall transient [6]. As far as fast power switches are concerned, e.g., GaN transistors, research has focused on open-loop

ISBN 978-80-261-0973-0, (C) University of West Bohemia, 2021

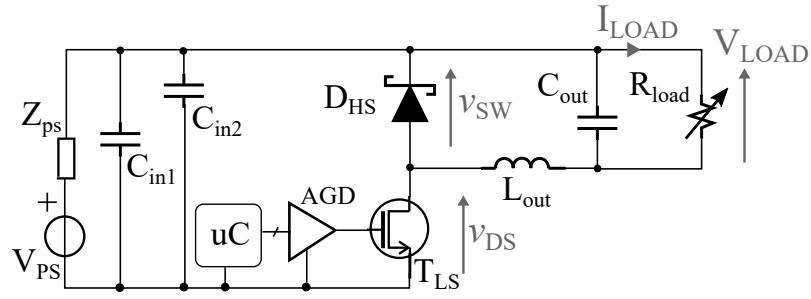

Fig. 1. Circuit schematic of the Buck converter exploited as case study.

AGDs, as they do not require high-bandwidth sensing circuit to measure peaks and slopes, nor fast digital circuits to evaluate the new profile on the basis of some algorithm. However, they may result in oscillating waveforms when the operating conditions are not constant, as reported in [7]. More precisely, variations of the load current should be careful considered, as they are rather common in practical scenarios. Reference [8] suggests to reduce the clock frequency of the proposed AGD when the load current increases. In [7], both current and temperature variations were investigated, and, a look-up table is proposed to store the AGD profiles for different currents in [9]. However, none of these works provides a method to adapt the AGD modulation profile to load current variations. Indeed, this issue deserves further investigations as it is crucial for the practical use of open-loop AGDs. The aim of this paper is to provide an experimental relation between the AGD parameters and the actual load current, in such a way, the nominal profile can be adapted to different loads without the need of a large memory to store the driving patterns or of devoting several steps to find the optimal profile, as discussed in [5].

The remainder of the paper is organized as follows. In Section II, the prototyped Buck converter featuring an AGD, which is later exploited as case study, is presented, then, the experimental setup and the effects of different load currents on switching waveforms are discussed in Section III. An effective tuning technique is presented in Section IV, and it is compared against the use of a fixed profile. Concluding remarks are drawn in Section V.

\section{THE CASE STUDY}

A low-side asynchronous Buck converter, like the one shown in Fig. 1, was exploited as case study in what 


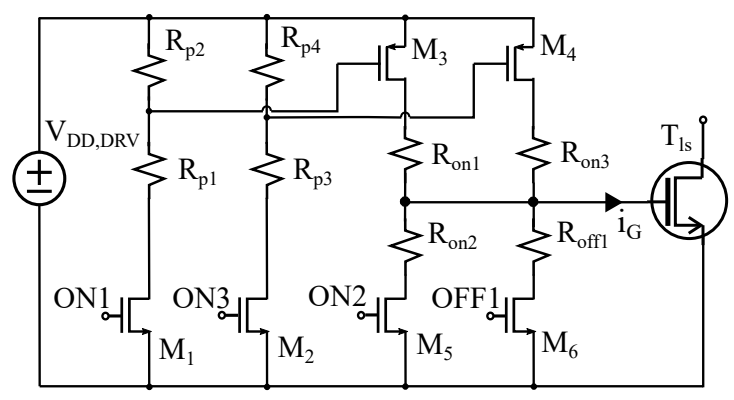

Fig. 2. The schematic of the prototyped AGD.

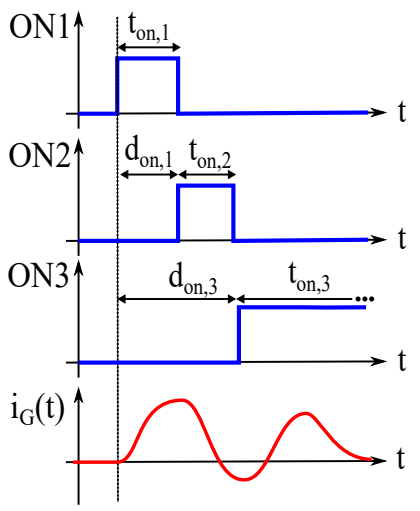

Fig. 3. The timing of the AGD control signals provided by the microcontroller.

follows. The power switch $T_{\mathrm{ls}}$ turns-on and turns-off with a fixed 0.25 duty cycle by means of the prototyped AGD shown in Fig. 2. The free-wheeling diode $D_{\text {hs }}$ let the current flowing in $L_{\text {out }}$ to discharge when $T_{\text {ls }}$ is off. Input $\left(C_{\text {in,1-2 }}\right)$ and output $\left(C_{\text {out }}\right)$ capacitors have been included to reduce the ripple affecting input and output voltages. Under nominal conditions, the converter steps down a $24 \mathrm{~V}$ input voltage $\left(V_{\mathrm{PS}}\right)$ to a $6 \mathrm{~V}$ output voltage $\left(V_{\mathrm{LOAD}}\right)$, while providing the load with a 0.5 A output current $\left(I_{\mathrm{LOAD}}\right)$. The nominal values of active and passive components exploited are listed in Table I.

The $T_{\mathrm{ls}}$ turn-on and the turn-off is achieved by means of the AGD shown in Fig. 2, which consists of discrete level components entirely. Such a driver is able either to turn-on the power transistor sharply or by modulating the gate current $i_{\mathrm{G}}$, depending on the control signals $O N 1, O N 2, O N 3$. Referring to Fig. 3, $O N 1, O N 2, O N 3$ are Pulse-Width-Modulated (PWM) outputs of the on-board microcontroller, whose duty cycles and phases can be interdependently adjusted by the firmware. The $M_{1-2}, M_{5-6}$ transistors were chosen to be in deep triode when the PWM signals are high. As far as the $T_{\mathrm{ls}}$ turn-on is concerned, when $O N 1$ is activated, $M_{1}$ and $M_{3}$ turn-on, thus the gate terminal of $T_{\mathrm{ls}}$ is connected to $V_{D D, D R V}$ through $R_{\mathrm{on} 1}$. When $T_{\text {ls }}$ reaches the Miller's plateau, $O N 1$ is deactivated, meaning that $M_{1}$ and $M_{3}$ turn-off and $O N 2$ is activated. As a consequence, some current is sunk by the $T_{\mathrm{ls}}$ gate and the power transistor is slowed down, resulting in an increase of the $T_{\mathrm{ls}}$ drain-source voltage $\left(v_{\mathrm{DS}}\right)$. Finally, $M_{5}$ is turned-off and $O N 3$ is activated, so $T_{\mathrm{ls}}$ can entirely turns-on, eventually. As depicted in Fig. 3, the duty cycles of $O N 1, O N 2, O N 3$ are named $t_{\mathrm{on}, 1-3}$, and the $O N 2, O N 3$ delays with respect to $O N 1$ are called
TABLE I

PARAMETER VALUES OF THE DESIGNED CONVERTER.

\begin{tabular}{cc|cc|cc}
\hline \hline Parameter & Value & Parameter & Value & Parameter & Value \\
\hline$C_{\text {in }, 1}$ & $100 \mu \mathrm{F}$ & $V_{\mathrm{LOAD}}$ & $6 \mathrm{~V}$ & $V_{\mathrm{PS}}$ & $24 \mathrm{~V}$ \\
$C_{\text {in }, 2}$ & $2.2 \mu \mathrm{F}$ & $R_{\text {load }}$ & $12 \Omega$ & $L_{\text {out }}$ & $220 \mu \mathrm{H}$ \\
$T_{\mathrm{ls}}$ & {$[11]$} & $R_{\mathrm{p} 1}$ & $20 \Omega$ & $R_{\mathrm{p} 2}$ & $28 \Omega$ \\
$R_{\mathrm{p} 3}$ & $39 \Omega$ & $R_{\mathrm{p} 4}$ & $56 \Omega$ & $R_{\text {on } 1}, R_{\text {on } 3}$ & $4.7 \Omega$ \\
$\mathrm{R}_{\text {on2 } 2}, \mathrm{R}_{\text {off } 1}$ & $0 \Omega$ & $V_{\mathrm{DD}, \mathrm{DRV}}$ & $10 \mathrm{~V}$ & $I_{\mathrm{LOAD}, \max }$ & $5 \mathrm{~A}$ \\
\hline \hline
\end{tabular}

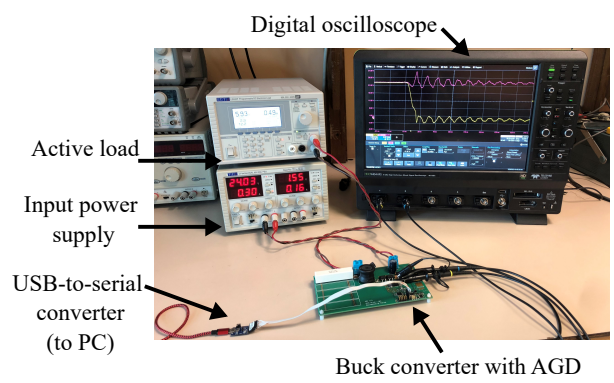

Fig. 4. Photograph of the experimental setup.

$d_{\mathrm{on}, 2-3}$, respectively. By exploiting the proposed circuit, it is possible to generate an $i_{\mathrm{G}}(t)$ current profile similar to that discussed in [10], which was found to damp the turn-on oscillations. The $T_{\mathrm{ls}}$ turn-off is always achieved by a single level, i.e., only $O F F 1$ is exploited, as the oscillations affecting such transient are negligible.

\section{EXPERIMENTAL SETUP}

Aiming to modify the AGD parameters, i.e., $t_{\mathrm{on}, 1-3}$ and $d_{\mathrm{on}, 2-3}$, automatically and to assess their impacts on switching waveforms, the experimental setup shown in Fig. 4 was exploited. The prototyped converter is supplied by a $24 \mathrm{~V}$ DC input voltage, and the resistor $R_{\text {load }}$ is emulated by an active load, which allows one to modify the output current $I_{\mathrm{LOAD}}$. The converter is provided with a serial interface, thereby the AGD parameters can be modified on-the-fly by a proper software running on the PC. In such a way, the corresponding switching waveforms acquired by the digital oscilloscope can be saved synchronously with the actual values of AGD parameters. As previously mentioned, the developed AGD can also operate as a Conventional Gate Driver (CGD) if $O N 1$ only is activated for the entire on-time of $T_{\mathrm{ls}}$. In such a case, and with the converter providing $0.5 \mathrm{~A}$ to the load, the corresponding $v_{\mathrm{DS}}$ voltage is shown in Fig. 5(a) by dashed line. It is affected by oscillations at $30 \mathrm{MHz}$ due to the parasitic resonant circuit excited during the transient, which result in the peak affecting the amplitude of the $v_{\mathrm{DS}}$ frequency spectrum, as shown in 5(b). Such oscillation does not only impact on the EMI of the converter, but also causes over-current and over-voltage on $i_{\mathrm{SW}}$ and $v_{\mathrm{SW}}$, eventually.

Then, the AGD parameters were manually tuned to account for the AGD propagation delays also, resulting in the $v_{\text {DS }}$ shown in Fig. 5(a) by solid line. The unwanted oscillations were entirely damped, thanks to a local increase of $v_{\mathrm{DS}}$ caused by the $O N 2$ activation. Indeed, the frequency spectrum of $v_{\mathrm{DS}}$ is no longer affected by the peak at $30 \mathrm{MHz}$, as shown in Fig. 5(b), meaning that a tuned AGD is effective in reducing the delivered EMI. The AGD benefits come at the cost of a slightly decrease 

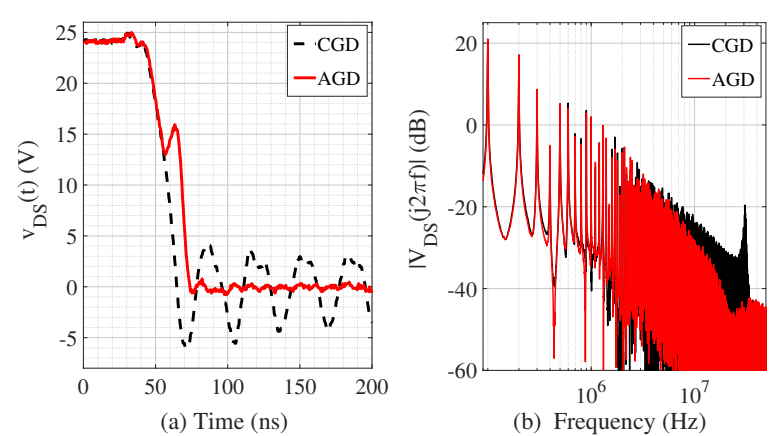

(b) Frequency $(\mathrm{Hz})$

Fig. 5. The drain-source voltage $v_{\mathrm{DS}}$ (a) with the power transistor driven sharply (dashed line) and by the tuned AGD (solid line) for a 0.5 load current acquired by the oscilloscope, (b) the corresponding frequency spectra.

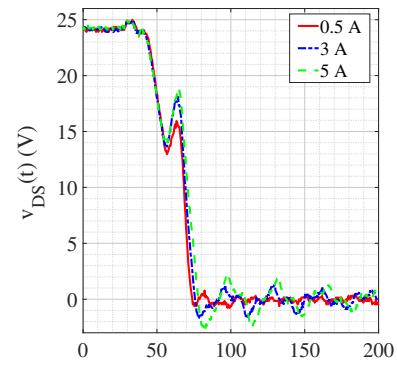

(a) Time (ns)

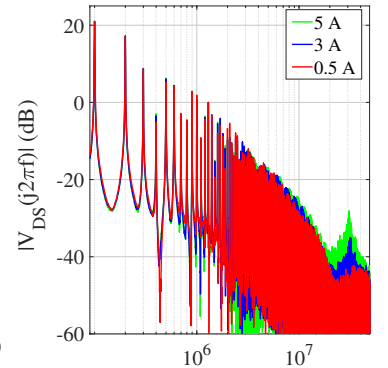

(b) Frequency $(\mathrm{Hz})$
Fig. 6. The drain-source voltage $v_{\mathrm{DS}}$ with (a) the AGD parameters are fixed and the current is increased to $3 \mathrm{~A}$ (dotted) and to $5 \mathrm{~A}$ (dashed line). In (b), the corresponding frequency spectra.

in the converter efficiency, as it reduces from $83.3 \%$ (CGD) to $82.7 \%$ (AGD). However, it should be noticed that AGDs outperform traditional solutions in terms of switching power losses [6]. It was set $t_{\text {on } 1, \text { nom }}=16 \mathrm{ns,}$ $d_{\mathrm{on} 2, \mathrm{nom}}=12.5 \mathrm{~ns}, t_{\mathrm{on} 2, \mathrm{nom}}=15.25 \mathrm{~ns}$ and $d_{\mathrm{on} 3, \mathrm{nom}}=17$ ns. On the contrary, the actual value of $t_{\text {on3,nom }}$ is only related to the $T_{\mathrm{ls}}$ on-time, thus it does not impact on switching performance.

With the load current increased whilst keeping constant the AGD parameters to their nominal values, the resulting $v_{\mathrm{DS}}$ are shown in Fig. 6(a) in the case of $I_{\mathrm{LOAD}}=3 \mathrm{~A}$ (dotted line), $I_{\mathrm{LOAD}}=5 \mathrm{~A}$ (dashed line), as well as $I_{\mathrm{LOAD}}=0.5 \mathrm{~A}$ (solid line) as a reference. The amplitude of oscillations increases, as well as the 30 $\mathrm{MHz}$ peak in the frequency spectra, as shown in Fig. $6(\mathrm{~b})$, which at $5 \mathrm{~A}$ output current is $10 \mathrm{~dB}$ higher than in nominal condition.

\section{Tuning of AGD Parameters}

In order to address such an issue, the proposed setup was used to modify the AGD parameters under different load currents to adapt the original profile, i.e., the one with the nominal parameters, to $I_{\mathrm{LOAD}}$ variations. The results of such investigations are reported in the remainder of the paper.

\section{A. Stretch of ON1}

As far as the $T_{\mathrm{ls}}$ turn-on is concerned, it results that oscillations are triggered by the $v_{\mathrm{SW}}$ rising edge, which takes place after the current in the power transistor $\left(i_{\mathrm{SW}}\right)$ has reached $I_{\mathrm{LOAD}}[12]$. Indeed, the $i_{\mathrm{SW}}$ slope is limited by the parasitic inductance of the power loop and by the input voltage, meaning that the higher $I_{\mathrm{LOAD}}$, the longer

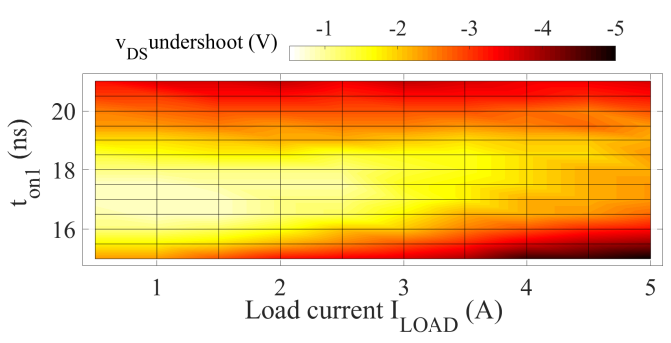

Fig. 7. Color-map representing the $v_{\mathrm{DS}}$ undershoot for [0.5,5] A load current and with the AGD parameters modified in according to (1).

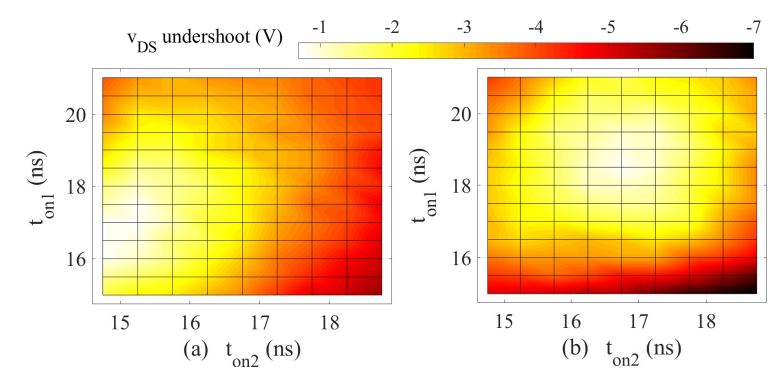

Fig. 8. Color-map representing the $v_{\mathrm{DS}}$ undershoot for (a) $0.5 \mathrm{~A}$ and (b) $5 \mathrm{~A}$ load current when both $t_{\mathrm{on}, 1}$ and $t_{\mathrm{on}, 2}$ are modified.

$T_{\text {ls }}$ will take to reach $I_{\mathrm{LOAD}}$. Thus, it seems reasonable to increase $t_{\mathrm{on}, 1}$ as the output current increases and to delay $O N 2, O N 3$ in the meanwhile. The AGD parameters were modified as

$$
\begin{array}{ll}
t_{\mathrm{on} 1}=t_{\mathrm{on} 1, \mathrm{nom}}+\Delta_{1} & d_{\mathrm{on} 2}=d_{\mathrm{on} 2, \mathrm{nom}}+\Delta_{1} \\
t_{\mathrm{on} 2}=t_{\mathrm{on} 2, \mathrm{nom}} & d_{\mathrm{on} 3}=d_{\mathrm{on} 3, \mathrm{nom}}+\Delta_{1}
\end{array}
$$

where $\Delta_{1}=[-1,5] \mathrm{ns}$ with a $0.5 \mathrm{~ns}$ step and the load current was varied in the $[0.5,5]$ A range. The colormap shown in Fig. 7 reports the $v_{\mathrm{DS}}$ undershoot for each configuration, more precisely, a darker color represents a higher undershoot. By increasing $t_{\mathrm{on}, 1}$ to $19 \mathrm{~ns}$ for a $5 \mathrm{~A}$ load current, the undershoot is equal to $2.2 \mathrm{~V}$, which is lower than the one with $t_{\text {on, } 1, \text { nom }}(3.2 \mathrm{~V})$. However, by stretching $t_{\mathrm{on}, 1}$ only, oscillations are not entirely damped.

\section{B. Stretch of ON1 and ON2}

Besides the $t_{\mathrm{on}, 1}$ increase, which resulted in few advantages, also $t_{\mathrm{on}, 2}$ was modified. As the load current increases, then also the energy stored in the parasitic inductance of the power loop increases, thus the power transistor has to dissipate more power to damp the oscillations. As previously discussed in Sect. II, ON2 controls the amount of gate charge sunk when $T_{\mathrm{ls}}$ is in Miller region, meaning that the longer $O N 2$ lasts, the more $v_{\mathrm{DS}}$ increases. With these considerations, both $t_{\mathrm{on}, 1}$ and $t_{\mathrm{on}, 2}$ were modified as

$$
\begin{array}{ll}
t_{\mathrm{on} 1}=t_{\mathrm{on} 1, \mathrm{nom}}+\Delta_{1} & d_{\mathrm{on} 2}=d_{\mathrm{on} 2, \mathrm{nom}}+\Delta_{1} \\
t_{\mathrm{on} 2}=t_{\mathrm{on} 2, \mathrm{nom}}+\Delta_{2} & d_{\mathrm{on} 3}=d_{\mathrm{on} 3, \mathrm{nom}}+\Delta_{1}+\Delta_{2}
\end{array}
$$

with $\Delta_{1}, \Delta_{2}$ in the $[-1,5]$ ns and $[-0.5,3.5]$ ns ranges, respectively. Figure 8(a) shows a color-map of the $v_{\mathrm{DS}}$ undershoot for $I_{\mathrm{LOAD}}=0.5 \mathrm{~A}$, Fig. 8 (b) for $I_{\mathrm{LOAD}}=5 \mathrm{~A}$. As it can be noticed, the lightest region, i.e., the one with minimum undershoot, has moved to higher $t_{\mathrm{on}, 1}$ and $t_{\mathrm{on}, 2}$, thus assessing the theoretical motivations reported. Indeed, by modifying both parameters, the undershoot can be effectively reduced. 


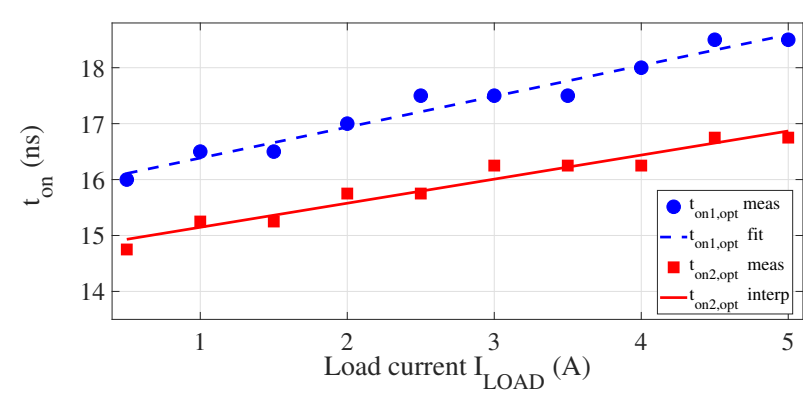

Fig. 9. Optimal values of $t_{\mathrm{on}, 1}$ (circle markers) and $t_{\mathrm{on}, 2}$ (square markers) obtained from experimental data, and the corresponding linear fit in dashed and solid line, respectively.
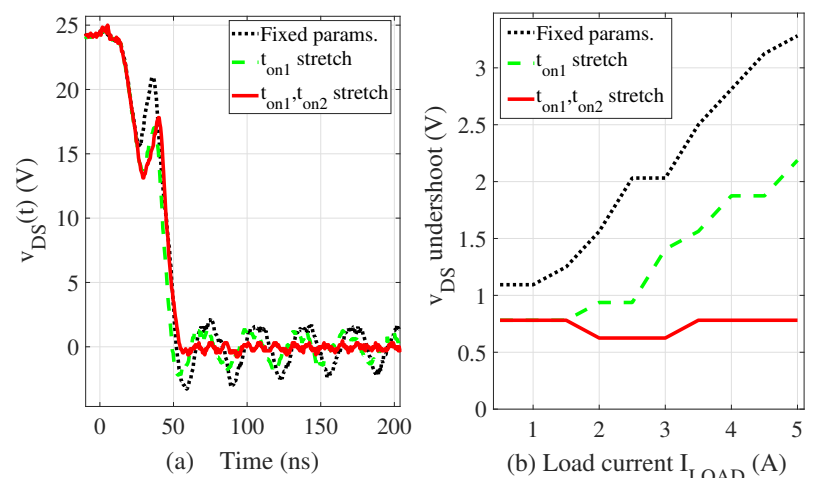

(b) Load current $\mathrm{I}_{\text {LOAD }}$ (A)

Fig. 10. In (a) the drain-source voltage with $5 \mathrm{~A}$ load current, in (b) the $v_{\text {DS }}$ undershoot. Dotted lines refer to fixed AGD parameters, dashed lines to $t_{\mathrm{on}, 1}$ stretch, and solid lines to $t_{\mathrm{on}, 1}$ and $t_{\mathrm{on}, 2}$ stretch.

In order to find a relation between the AGD parameters and the load current, the optimal $t_{\mathrm{on}, 1}, t_{\mathrm{on}, 2}$ values related to the minimum undershoot were extrapolated for each current, and plotted in Fig. 9 by circle and square markers, respectively. Then, a linear fit was performed resulting in the dashed and solid curves shown in the same plot, which well fit the experimental data. The resulting expressions were found to be equal to

$$
t_{\mathrm{on}, 1}=p_{1} I_{\mathrm{LOAD}}+p_{0} \quad t_{\mathrm{on}, 2}=p_{3} I_{\mathrm{LOAD}}+p_{2}
$$

where $p_{0}=15.8 \mathrm{~ns}, p_{1}=0.55 \mathrm{~ns} / \mathrm{A}, p_{2}=14.7 \mathrm{~ns}$ and $p_{3}=0.43 \mathrm{~ns} / \mathrm{A}$. This point is crucial, as it states that the gate current profile, obtained under a given load current, can be adapted to different load currents without affecting the undershoot. Indeed, only four coefficients are needed to be stored, while the computation of the new parameters required sums and products only.

\section{Comparison of the analyzed techniques}

Finally, a comparison between the analyzed techniques, i.e., fixed profile, $O N 1$ only and $O N 1, O N 2$ stretch, is presented. For a load current of $5 \mathrm{~A}$, the configuration that minimize the undershoot was selected for each technique, and the corresponding $v_{\mathrm{DS}}$ shown in Fig. 10(a). With the AGD parameters kept fixed, oscillations are superimposed onto $v_{\mathrm{DS}}$, as shown by the dotted line. If $t_{\mathrm{on}, 1}$ is increased, they are mitigated but they are still not negligible (dashed line), on the contrary, when both $t_{\mathrm{on}, 1}$ and $t_{\mathrm{On} 21}$ increase proportionally to $I_{\mathrm{LOAD}}$, then $v_{\mathrm{DS}}$ is not affected by oscillations (solid line). Finally, the undershoot was measured for each technique and plotted in Fig. 10(b). For the [0.5,5] A current range, only the third technique allows the AGD to keep the undershoot to its minimum value (solid line), whether in case of fixed profile (dotted line) or by changing $t_{\mathrm{on}, 1}$ only (dashed line) the undershoot increases.

\section{CONCLUSiON}

As far as fast power switches are concerned, openloop AGDs are a viable solution, however, they suffer from suboptimal tuning when the load current changes. This paper investigates such an issue on a prototyped Buck converter, in which the power transistor is driven by an AGD, capable of modulating the gate current profile. Experimental results assessed the AGD under nominal conditions, and they pointed out its lack of effect when the operating conditions are modified. It was found that the undershoot affecting the drain-source voltage is effectively controlled if the timing parameters of the AGD varies proportionally with the load current.

\section{ACKNOWLEDGMenT}

This work was partially supported by the Power Electronics Innovation Center (PEIC), Politecnico di Torino, Italy.

\section{REFERENCES}

[1] PELS members, "International Technology Roadmap for Wide Bandgap Power Semiconductors", IEEE Power Electronics Society, 2019.

[2] A. Bhargava, D. Pommerenke, K. W. Kam, F. Centola and C. W. Lam, "DC-DC Buck Converter EMI Reduction Using PCB Layout Modification," in IEEE Transactions on Electromagnetic Compatibility, vol. 53, no. 3, pp. 806-813, Aug. 2011.

[3] E. A. Jones, F. F. Wang and D. Costinett, "Review of Commercial GaN Power Devices and GaN-Based Converter Design Challenges," in IEEE Journal of Emerging and Selected Topics in Power Electronics, vol. 4, no. 3, pp. 707-719, Sept. 2016.

[4] H. Ghorbani, V. Sala, A. Paredes and L. Romeral, "A novel EMI reduction design technique in IGBT gate driver for turnon switching mode," 2016 18th European Conference on Power Electronics and Applications (EPE'16 ECCE Europe), Karlsruhe, 2016, pp. 1-7.

[5] D. J. Rogers and B. Murmann, "Digital Active Gate Drives using sequential optimization," 2016 IEEE Applied Power Electronics Conference and Exposition (APEC), Long Beach, CA, USA, 2016, pp. 1650-1656.

[6] A. P. Camacho, V. Sala, H. Ghorbani and J. L. R. Martinez, ”A Novel Active Gate Driver for Improving SiC MOSFET Switching Trajectory," in IEEE Transactions on Industrial Electronics, vol. 64, no. 11, pp. 9032-9042, Nov. 2017.

[7] T. Sai et al., "Load Current and Temperature Dependent Optimization of Active Gate Driving Vectors," 2019 IEEE Energy Conversion Congress and Exposition (ECCE), Baltimore, MD, USA, 2019, pp. 3292-3297.

[8] J. J. O. Dalton et al., "Stretching in Time of GaN Active Gate Driving Profiles to Adapt to Changing Load Current," 2018 IEEE Energy Conversion Congress and Exposition (ECCE), Portland, OR, 2018, pp. 3497-3502.

[9] F. Zhang, X. Yang, Y. Ren, L. Feng, W. Chen and Y. Pei, "Advanced Active Gate Drive for Switching Performance Improvement and Overvoltage Protection of High-Power IGBTs," in IEEE Transactions on Power Electronics, vol. 33, no. 5, pp. 3802-3815, May 2018.

[10] M. V. Quitadamo, E. Raviola and F. Fiori, "Investigation on the Switching Waveforms of GaN Power Devices to Gate Current Profiles," 2019 International Conference on Power Electronics, Control and Automation (ICPECA), New Delhi, India, 2019, pp. $1-6$.

[11] "IPB065N15N3 Datasheet", [Online] https://www.infineon. com/dgdl/Infineon-IPB065N15N3G-DS-v02_01-en.pdf?fileId= db3a30432662379201266a0379d1225c, Accessed on: Dec.28, 2020.

[12] M. V. Quitadamo, E. Raviola and F. Fiori, "A Criterion for an Optimal Switching of Power Transistors," 2019 12th International Workshop on the Electromagnetic Compatibility of Integrated Circuits (EMC Compo), Hangzhou, China, 2019, pp. 174-176. 